\title{
TWO NEW DECOMPOSITION ALGORITHMS FOR TRAINING BOUND-CONSTRAINED SUPPORT VECTOR MACHINES*
}

\author{
Lingfeng Niu *, Ruizhi Zhou *, Xi Zhao *, Yong Shi *
}

\begin{abstract}
Bound-constrained Support Vector Machine(SVM) is one of the stateof-art model for binary classification. The decomposition method is currently one of the major methods for training SVMs, especially when the nonlinear kernel is used. In this paper, we proposed two new decomposition algorithms for training bound-constrained SVMs. Projected gradient algorithm and interior point method are combined together to solve the quadratic subproblem efficiently. The main difference between the two algorithms is the way of choosing working set. The first one only uses first order derivative information of the model for simplicity. The second one incorporate part of second order information into the process of working set selection, besides the gradient. Both algorithms are proved to be global convergent in theory. New algorithms is compared with the famous package BSVM. Numerical experiments on several public data sets validate the efficiency of the proposed methods.
\end{abstract}

Keywords: decomposition algorithm, support vector machine, quadratic programming, global convergence

\section{Introduction}

Let $S=\left\{\left(\mathbf{x}_{1}, y_{1}\right), \cdots,\left(\mathbf{x}_{l}, y_{l}\right)\right\}$ be a set of training samples belonging to $m$ different classes, where $\mathbf{x}_{i} \in X \subseteq \Re^{n}$ and $y_{i} \in Y$ are the input data and corresponding label for sample $i$, respectively. A classification problem can be interpreted as finding a classifier, which from mathematical point of view is a function that maps an instance

${ }^{*}$ Part of this work has been presented at the IEEE/WIC/ACM International Conference on Web Intelligence special session on "complex methods for data and web mining", 12 August 2014, Warsaw, Poland.

${ }^{*}$ Research Center on Fictitious Economy \& Data Science, University of Chinese Academy of Sciences, Key Laboratory of Big Data Mining and Knowledge Management, Chinese Academy of Sciences, Beijing, China 
$\mathbf{x}$ into an element $y$ of $Y$. When there are only two labels in the label set $Y$, the problem is called binary classification. When the possible labels are more than two, the problem is called multi-class classification. We will concentrate on the binary classification problem in this paper.

Support Vector Machine (SVM) $[2,4,20,21]$ is one of the state-of-the-art classifiers nowadays. Subsequently, different improved versions have been proposed [31, 30, 29]. The primal model of the standard SVM is formularized as the following quadratic programmming $(\mathrm{QP})$

$$
\begin{array}{ccc}
\min _{\mathbf{w}, b, \xi} & \frac{1}{2}\|\mathbf{w}\|^{2}+C \sum_{i=1}^{l} \xi_{i} & \\
\text { s.t. } & y_{i}\left(\mathbf{w}^{T} \boldsymbol{\phi}\left(\mathbf{x}_{i}\right)+b\right) \geq 1-\xi_{i}, & \forall i=1, \cdots, l, \\
& \xi_{i} \geq 0 & \forall i=1, \cdots, l,
\end{array}
$$

where, $C$ is a pre-given model parameter, $\phi(\cdot)$ is the mapping from input space to the feature space, which may be infinite dimension. The dual form of (1) can be written as follow, which is always a finite QP:

$$
\begin{array}{cc}
\min _{\boldsymbol{\alpha}} & f(\boldsymbol{\alpha})=\frac{1}{2} \boldsymbol{\alpha}^{T} \hat{Q} \boldsymbol{\alpha}^{T}-\mathbf{1}^{T} \boldsymbol{\alpha} \\
\text { s.t. } & \boldsymbol{y}^{T} \boldsymbol{\alpha}=0, \\
& \mathbf{0} \leq \boldsymbol{\alpha} \leq \mathbf{C},
\end{array}
$$

where, $\mathbf{0}$ and $\mathbf{1}$ represent the vector of all zeros and ones, respectively, $\mathbf{C}=C \mathbf{1}$, $\hat{Q}$ is an $l$ by $l$ matrix, $\hat{Q}_{i, j} \equiv y_{i} y_{j} K\left(\mathbf{x}_{i}, \mathbf{x}_{j}\right)$, and $K\left(\mathbf{x}_{i}, \mathbf{x}_{j}\right) \equiv \phi\left(\mathbf{x}_{i}\right)^{T} \boldsymbol{\phi}\left(\mathbf{x}_{j}\right)$ is the kernel function. When $K(\cdot, \cdot)$ satisfies Mercer condition [16], matrix $\hat{Q}$ is positive semidefinite.

In this paper, we consider a simple modification model of the standard SVM (1) as follow,

$$
\begin{array}{cll}
\min _{\mathbf{w}, b, \xi} & \frac{1}{2}\left(\|\mathbf{w}\|^{2}+b^{2}\right)+C \sum_{i=1}^{l} \xi_{i} & \\
\text { s.t. } & y_{i}\left(\mathbf{w}^{T} \phi\left(\mathbf{x}_{i}\right)+b\right) \geq 1-\xi_{i}, & \forall i=1, \cdots, l, \\
& \xi_{i} \geq 0 & \forall i=1, \cdots, l,
\end{array}
$$

The dual form of the above problem is the following QP, which contains bound constraints only:

$$
\begin{array}{cc}
\min _{\boldsymbol{\alpha}} & f(\boldsymbol{\alpha})=\frac{1}{2} \boldsymbol{\alpha}^{T} Q \boldsymbol{\alpha}^{T}-\mathbf{1}^{T} \boldsymbol{\alpha} \\
\text { s.t. } & \mathbf{0} \leq \boldsymbol{\alpha} \leq \mathbf{C}
\end{array}
$$

where, $Q_{i, j} \equiv y_{i} y_{j}\left(K\left(\mathbf{x}_{i}, \mathbf{x}_{j}\right)+1\right)$. Suppose the optimal solution of problem (4) is $\alpha^{*}$. Then the classifier can be written as

$$
H(\mathbf{x})=\operatorname{sign}\left\{\sum_{i=1}^{l} \alpha_{i}^{*} y_{i}\left(K\left(\mathbf{x}, \mathbf{x}_{i}\right)+1\right)\right\} .
$$


Similar to the standard SVM, if $\alpha_{i}^{*} \neq 0$, the corresponding sample $i$ is called a Support Vector(SV). Furthermore, if $\alpha_{i}^{*}=C$, it is called a Bounded Support Vector(BSV). To show the difference with the standard SVM, (3) and (4) are usually called the bound-constrained SVMs.

Bound-constrained SVMs are once proposed independently by Fireß, Cristianini and Campbell in [19], Mangasarian and Musicant in [14] and [15]. Mangasarian and Musicant [14] proves that for the linear kernel case, there exist some kind of equivalence between bound-constrained SVMs and the standard SVMs. The result can be concluded in the following proposition.

Proposition 1. [14] For the bound-constrained SVMs with linear kernel function $k(\mathbf{x}, \mathbf{y})$, Suppose $(\mathbf{w}, b, \xi)$ is an arbitrary solution of problem (3). If any solution $\hat{\mathbf{v}}$ of the following system:

$$
A^{T} v=0, \mathbf{1}^{T} v=b, \mathbf{v} \geq 0
$$

satisfies

$$
\mathbf{1}^{T} \hat{\mathbf{v}}\left(\mathbf{1}^{T} \xi-1\right) \leq b^{2} .
$$

There must exists a sufficient large $C$, such that $(\mathbf{w}, b, \xi)$ is also a solution of the standard primal SVM model (1), where $A^{T}=\left(x_{1}, \cdots, x_{l}\right) \in \Re^{n \times l}$, i.e. each row of of $A$ is the feature vector of a sample.

Because of the simple formulation and good classification performance of boundconstrained SVM, it draws a lot of attention in the recent years. For the linear kernel case, Mangasarian and Musicant proposed to solve the model (4) by the overrelaxation method in [14]. They proved the global convergence and the linear convergent rate of the algorithm at the same time. Hsieh et al gave a novel dual coordinate descent method and proved the algorithm reaches an $\epsilon$-accurate solution in $O(\log (1 / \epsilon))$ iterations in [8]. Several researchers also explore how to train the primal form of (4) and the extended models fast. The existing algorithms can be broadly categorized into two categories: the cutting-plane methods[11, 5, 12, 13, 25], and subgradient methods[3, 17]. For example, in [17], Shalev-Shwartz et al described and analyzed a simple and effective stochastic sub-gradient descent algorithm and prove that the number of iterations required to obtain a solution of accuracy $\epsilon$ is $O(1 / \epsilon)$. Generally speaking, without counting the loading time, these recent advances on linear classification have shown that training one million instances takes only a few seconds $[22]$.

For the general nonlinear kernel case, the scale of the problems grows quadratically with the number of samples $l$ due to the appearance of kernel matrix. Therefore, for the middle and large scale problem, decomposition techniques are needed to handle the problems arising from the limitation of the memory. Hsu and Lin analyzed in thorough how to design a decomposition algorithm for problem (4) in [9], which mainly discussed how to design an effective working set selection rule based on the existing successful working set selection rule for standard SVMs. They started from applying the working set selection strategy of $S V M^{\text {light }}$ [10] to model (4) directly. Step by step, a series of heuristic techniques were added to improve the performance of the selection rule. Different form the designation idea in [9], we will discuss how 
to derive effective working set selection rule from the optimization algorithm design point of view. Although the derivation is much more concise than [9], the numerical experiments in section 4 show that the computation efficiency of our new methods is at least as good as Hsu and Lin's method.

The structure of the paper is as follows. In Section 2, after introducing the general decomposition algorithm framework for problem (4), we propose our new working set selection rules. In section 3 , we prove that the decomposition algorithms based on our new working set selection strategies are globally convergent. In section 4, we concentrate on the efficient implementation. Numerical experiments on several public data sets are listed. A brief conclusion and some possible future works are given in the last section.

\section{The New Decomposition Algorithms}

\subsection{The decomposition algorithm framework}

Because $Q_{i j}$ is in general not zero, $Q$ becomes a fully dense matrix. Due to the density of $Q$, a prohibitive amount of memory is required to store the matrix. Thus traditional optimization algorithms, which needs the whole Hessian matrix of objective function, cannot be directly used. To conquer this difficulty, several researchers (for example, Osuna et al [24], Platt[26], Joachims[27], Saunders et al[28]) have proposed decomposition methods. The key idea of decomposition is to update a small part of variables at each iteration, and to solve a sequence of constant-size problems. Then, the solution of a large-scale dense problem can be found by solving a number of small problems.

Now we denote the gradient of objective function (4a) as:

$$
F(\boldsymbol{\alpha})=\left(\frac{\partial f(\boldsymbol{\alpha})}{\partial \alpha_{i}}\right)=Q \boldsymbol{\alpha}-\mathbf{1}
$$

for any $i \in\{1, \cdots, l\}$, the $i$-th element of $F(\boldsymbol{\alpha})$ is:

$$
F_{i}(\boldsymbol{\alpha})=\frac{\partial f(\boldsymbol{\alpha})}{\partial \alpha_{i}}=\sum_{j=1}^{l} Q_{i j} \alpha_{j}-1
$$

The index set of variables optimized at a current iteration is called the working set and denoted as $\mathcal{B}$. Let $\mathcal{N} \equiv\{1, \cdots, l\} / \mathcal{B}$, and superscript record the iteration number. Using the notation above, the general decomposition method for problem (4) is described in Algorithm 1. Notice that Algorithm 1 is just a framework, to make it be implemented in practice, the specific working set selection rule and QP subproblem solver (8) should be given. In the rest part of this section, we will discuss how to select the working set effectively from the optimization design point of view. 
Denote $Q \operatorname{sub}\left(\boldsymbol{\alpha}^{(k)}, \mathcal{B}\right)$ as the optimal objective function value of $\mathrm{QP}$

$$
\begin{array}{ccc}
\min _{\boldsymbol{d}} & f\left(\boldsymbol{\alpha}^{(k)}+\boldsymbol{d}\right)-f^{(k)} & \\
\text { s.t. } & -\alpha_{i}^{(k)} \leq d_{i} \leq C-\alpha_{i}^{(k)} & \forall i \in \mathcal{B} ; \\
& d_{i}=0, & \forall i \notin \mathcal{B} .
\end{array}
$$

If only one iteration is considered, the best working set is $\operatorname{argmin}_{\mathcal{B}:|\mathcal{B}|=n_{B}} Q \operatorname{sub}\left(\boldsymbol{\alpha}^{(k)}, \mathcal{B}\right)$. However, this choice requires solving $\left(\begin{array}{c}l \\ n_{B}\end{array}\right)$ QPs $(7)$, which is too expensive to manipulate in practice. To decrease the computational cost, we will present two new working set selection methods in the next two subsections.

Algorithm 1 Decomposition Algorithm Framework for Bound-Constrained SVMs

Step 0. Initialization. Given the upper bound for the size of the workings set $n_{B} \geq 1$, the initial working set $\mathcal{B}^{(0)}$ and the initial point $\boldsymbol{\alpha}^{(0)}=\mathbf{0} \in \Re^{l}$. Set $k=0$.

Step 1. Subproblem Solution Construct and solve the quadratic subproblem

$$
\begin{array}{rc}
\min _{\left\{\alpha_{i}\right\}_{i \in \mathcal{B}^{(k)}}} & \frac{1}{2} \sum_{i, j \in \mathcal{B}^{(k)}} Q_{i j} \alpha_{i} \alpha_{j}+\sum_{i \in \mathcal{B}^{(k)}}\left(F_{i}^{(k)}-\sum_{j \in \mathcal{B}^{(k)}} Q_{i j} \alpha_{j}^{(k)}\right) \alpha_{i} \\
\text { s.t. } & 0 \leq \alpha_{i} \leq C, \quad \forall i \in \mathcal{B}^{(k)}
\end{array}
$$

Denote the optimal solution as $\left\{\bar{\alpha}_{i}\right\}_{i \in \mathcal{B}^{(k)}}$. Update the iteration point as

$$
\alpha_{i}^{(k+1)}=\left\{\begin{array}{cc}
\bar{\alpha}_{i} & \text { if } i \in \mathcal{B}^{(k)} \\
\alpha_{i}^{(k)} & \text { if } i \notin \mathcal{B}^{(k)}
\end{array}\right.
$$

Step 2. Gradient Update. Update gradient based on formula (5). Set $k:=k+1$. Step 3. Working Set Selection. Test whether the iterates should be terminated according to some stopping criteria. If not stop, select at most $n_{B}$ indices to form a new working set $\mathcal{B}^{(k)}$, and go back to Step 1. Otherwise, output $\alpha^{(k)}$ as the optimal solution and stop.

\subsection{Using first order information for working set selection}

A straightforward way of simplifying problem (7) is dropping the second order information in the objective function, and confine the incremental variables between 1 and -1 , which results in the following Linear Programming(LP):

$$
\begin{array}{ccc}
\min _{\left\{d_{i}\right\}_{i \in \mathcal{B}}} & \sum_{i \in \mathcal{B}} F_{i}^{(k)} d_{i} & \\
& -\alpha_{i}^{(k)} \leq d_{i} \leq C-\alpha_{i}^{(k)}, & \forall i \in \mathcal{B} ; \\
-1 \leq d_{i} \leq 1, & \forall i \in \mathcal{B} .
\end{array}
$$


Denote the optimal objective function value of problem $(12)$ as $\operatorname{Lsub}\left(\boldsymbol{\alpha}^{(k)}, \mathcal{B}\right)$. Then, $\operatorname{argmin}_{\mathcal{B}:|\mathcal{B}|=n_{B}} \operatorname{Lsub}\left(\boldsymbol{\alpha}^{(k)}, \mathcal{B}\right)$ should be a good choice as the working set $\mathcal{B}^{(k)}$. Dividing the constraints $(12 \mathrm{~b})$ and $(12 \mathrm{c})$ by samples, problem (12) can be solved as $|\mathcal{B}|$ independent LPs. In details, for any $i \in \mathcal{B}$,

$$
\begin{array}{cc}
\min _{d_{i}} & d_{i} F_{i}^{(k)} \\
\text { s.t. } & \max \left\{-1,-\alpha_{i}^{(k)}\right\} \leq d_{i} \leq \min \left\{1, C-\alpha_{i}^{(k)}\right\} .
\end{array}
$$

which solution can be represented as

$$
\hat{d}_{i}=\left\{\begin{aligned}
\max \left\{-\alpha_{i}^{(k)},-1\right\}, & \text { if } F_{i}^{(k)} \geq 0, \\
\min \left\{C-\alpha_{i}^{(k)}, 1\right\}, & \text { if } F_{i}^{(k)}<0,
\end{aligned}\right.
$$

The above formula can be rewritten as a more compact form:

$$
\hat{d}_{i}=-\operatorname{sign}\left(F_{i}^{(k)}\right) \min \left\{\frac{1}{2} C\left(1-\operatorname{sign}\left(F_{i}^{(k)}\right)\right)-\alpha_{i}^{(k)}, 1\right\} .
$$

Furthermore, the corresponding optimal objective function value of LP (10) can be represented as

$$
-\left|F_{i}^{(k)}\right| \min \left\{\frac{1}{2} C\left(1-\operatorname{sign}\left(F_{i}^{(k)}\right)\right)-\alpha_{i}^{(k)}, 1\right\} .
$$

Based on these discussion, for $\forall i=1, \cdots, l$, define

$$
\ell_{i}(\boldsymbol{\alpha})=-\left|F_{i}^{(k)}\right| \min \left\{\frac{1}{2} C\left(1-\operatorname{sign}\left(F_{i}^{(k)}\right)\right)-\alpha_{i}^{(k)}, 1\right\}
$$

namely,

$$
\ell_{i}(\boldsymbol{\alpha})=\left\{\begin{array}{cl}
\max \left\{-\alpha_{i}^{(k)},-1\right\} F_{i}(\alpha), & \text { if } F_{i}^{(k)} \geq 0 \\
\min \left\{C-\alpha_{i}^{(k)}, 1\right\} F_{i}(\alpha), & \text { if } F_{i}^{(k)}<0
\end{array}\right.
$$

Then,

$$
\operatorname{Lsub}\left(\boldsymbol{\alpha}^{(k)}, \mathcal{B}\right)=\sum_{i \in \mathcal{B}} \ell_{i}^{(k)}
$$

Therefore, instead of solving $\left(\begin{array}{c}l \\ n_{B}\end{array}\right)$ LPs (10), we can compute $\ell^{(k)}$, and select the indices corresponding to the $n_{B}$ smallest elements of $\ell_{i}^{(k)}$ directly to get the value of

$$
\min _{\mathcal{B}:|\mathcal{B}|=n_{B}} \operatorname{Lsub}\left(\boldsymbol{\alpha}^{(k)}, \mathcal{B}\right) .
$$

Especially, when $\boldsymbol{\ell}^{(k)}=\mathbf{0}$, no new working set can be selected, and the iterates can be terminated naturally. To summarize, we describe this simple working set selection rule in Algorithm 2. 
Algorithm 2 The First-Order Working Set Selection Rule

Compute $\boldsymbol{\ell}^{(k)}$ by formula (11).

if $\ell^{(k)} \neq \mathbf{0}$ then

Sort the indices set $\{1, \cdots, l\}$ by the values of $\ell^{(k)}$ in increasing order. Choose the first $n_{B}$ indices to form the working set $\mathcal{B}^{(k)}$.

else

Output $\boldsymbol{\alpha}^{(k)}$ as the optimal solution and terminate the iterates.

end if

In practice, we find that some indices enter and leave the working set lots of times when applying working set selection rule Algorithm 2 directly to Algorithm 1. This causes the decomposition algorithm converging very slow. In order to avoid the zigzagging phenomenon, we keep part of indices from previous working sets at each iteration. To be more precise, at most $n_{N}$ new indices are allowed to enter into the working set, where $1 \leq n_{N} \leq n_{B}$. Other indices are taken from the working set of last iteration. One thing we want to stress is that different techniques of inheriting indices has been used by several state-of-the-art solvers $[10,9,23]$. In this work, we use the inheriting strategy used in [23] to revise our working set selection rules we proposed before. Details are given in Algorithm 3.

Algorithm 3 The Practical First-Order Working Set Selection Rule

Compute $\boldsymbol{\ell}^{(k)}$ by (11).

if $\ell^{(k)} \neq \mathbf{0}$ then

i ) Set $\hat{\mathcal{B}}=\mathcal{B}^{(k)}$ and $\mathcal{B}^{(k+1)}=\emptyset$.

ii) Sort the indices set $\{1, \cdots, l\}$ by the values of $\ell^{(k)}$ in increasing order; Add the first $n_{N}$ indices to form the working set $\mathcal{B}^{(k+1)}$; Set $\hat{\mathcal{B}}:=\hat{\mathcal{B}} \backslash \mathcal{B}^{(k+1)}$.

iii) Fill $j \in\left\{i \mid i \in \hat{\mathcal{B}}, 0<\alpha_{i}^{(k)}<C\right\}$, which has the lowest number of consecutive iterations in working set, to $\mathcal{B}^{(k+1)}$ and remove $j$ from $\hat{\mathcal{B}}$ until $\left|\mathcal{B}^{(k+1)}\right|=n_{B}$ or there is no such element.

iv) Fill $j \in\left\{i \mid i \in \hat{\mathcal{B}}, \alpha_{i}^{(k)}=0\right\}$, which has the lowest number of consecutive iterations in working set, to $\mathcal{B}^{(k+1)}$ and remove it from $\hat{\mathcal{B}}$ until $\left|\mathcal{B}^{(k+1)}\right|=n_{B}$ or there is no such element.

v) Fill $j \in\left\{i \mid i \in \hat{\mathcal{B}}, \alpha_{i}^{(k)}=C\right\}$, which has the lowest number of consecutive iterations in working set to $\mathcal{B}^{(k+1)}$ and remove it from $\hat{\mathcal{B}}$ until $\left|\mathcal{B}^{(k+1)}\right|=n_{B}$ or there is no such element.

else

Output $\alpha^{(k)}$ as the optimal solution and terminate the iterations.

end if 


\subsection{Using second order information for working set selection}

In the algorithm described in the last subsection, only first-order information of the objective function (4a) is used for simplicity. However, this does not mean that there is no simple and feasible method which uses second order information for choosing working set. In this subsection, we will consider how to include second order information into the process of working set selection.

Besides keeping both the first order information, we incorporate the diagonal second order information, and get the following revised QP:

$$
\begin{array}{cl}
\min _{\left\{d_{i}\right\}_{i \in \mathcal{B}}} & \sum_{i \in \mathcal{B}}\left(F_{i}^{(k)} d_{i}+\frac{1}{2} Q_{i i} d_{i}^{2}\right) \\
& -\alpha_{i}^{(k)} \leq d_{i} \leq C-\alpha_{i}^{(k)}, \quad \forall i \in \mathcal{B} ; \\
-1 \leq d_{i} \leq 1, & \forall i \in \mathcal{B} .
\end{array}
$$

Denote the optimal objective function value of problem $(12)$ as $D Q \operatorname{sub}\left(\boldsymbol{\alpha}^{(k)}, \mathcal{B}\right)$. Since more information of the objective model is used, $\operatorname{argmin}_{\mathcal{B}:|\mathcal{B}|=n_{B}} D Q \operatorname{sub}\left(\boldsymbol{\alpha}^{(k)}, \mathcal{B}\right)$ should be a better choice of $\mathcal{B}^{(k)}$ than the set selection rule given in the last subsection.

Similar to the discussion of subsection 2.2, dividing the constraints (12b) and (12c) by samples, problem (12) can be solved as $|\mathcal{B}|$ independent QPs. In details, for any $i \in \mathcal{B}$,

$$
\begin{array}{cc}
\min _{d_{i}} & d_{i} F_{i}^{(k)}+\frac{1}{2} Q_{i i} d_{i}^{2} \\
\text { s.t. } & \max \left\{-1,-\alpha_{i}^{(k)}\right\} \leq d_{i} \leq \min \left\{1, C-\alpha_{i}^{(k)}\right\} .
\end{array}
$$

which solution can be represented as

$$
\hat{d}_{i}= \begin{cases}\max \left\{-\frac{F_{i}^{(k)}}{Q_{i i}}, \max \left\{-\alpha_{i}^{(k)},-1\right\}\right\}, & \text { if } F_{i}^{(k)} \geq 0, \\ \min \left\{-\frac{F_{i}^{(k)}}{Q_{i i}}, \min \left\{C-\alpha_{i}^{(k)}, 1\right\}\right\}, & \text { if } F_{i}^{(k)}<0\end{cases}
$$

The above formula can be rewritten as a more compact form:

$$
\hat{d}_{i}=\operatorname{mid}\left\{-\frac{F_{i}^{(k)}}{Q_{i i}}, \max \left\{-\alpha_{i}^{(k)},-1\right\}, \min \left\{C-\alpha_{i}^{(k)}, 1\right\}\right\} .
$$

Furthermore, the corresponding optimal objective function value of problem (13) can be represented as $F_{i}^{(k)} \hat{d}_{i}+\frac{1}{2} Q_{i i} \hat{d}_{i}^{2}$. Based on these discussion, for $\forall i=1, \cdots, l$, define

$$
\breve{d}_{i}(\boldsymbol{\alpha})=\operatorname{mid}\left\{-\frac{F_{i}(\boldsymbol{\alpha})}{Q_{i i}}, \max \left\{-\alpha_{i},-1\right\}, \min \left\{C-\alpha_{i}, 1\right\}\right\}
$$

and

$$
q_{i}(\boldsymbol{\alpha})=F(\boldsymbol{\alpha})_{i} \breve{d}_{i}(\boldsymbol{\alpha})+\frac{1}{2} Q_{i i} \breve{d}_{i}(\boldsymbol{\alpha})^{2} .
$$

Then, $D Q \operatorname{sub}\left(\boldsymbol{\alpha}^{(k)}, \mathcal{B}\right)=\sum_{i \in \mathcal{B}} q_{i}^{(k)}$. Therefore, instead of solving $\left(\begin{array}{c}l \\ n_{B}\end{array}\right)$ QPs (13), we can compute $\boldsymbol{q}^{(k)}$, and select the indices corresponding to the $n_{B}$ smallest elements 
of $q_{i}^{(k)}$ to form $\arg \min _{\mathcal{B}:|\mathcal{B}|=n_{B}} D Q \operatorname{sub}\left(\boldsymbol{\alpha}^{(k)}, \mathcal{B}\right)$ directly. Especially, when $\boldsymbol{q}^{(k)}=\mathbf{0}$, no new working set can be selected, and the iterates terminate naturally. Similar practical working set selection strategy can also be used here to avoid the zigzagging phenomenon. To summarize, we describe the new working set selection rule in Algorithm 4.

\section{Global Convergence Analysis}

In this section, we will prove that the decomposition algorithms based on our new working set selection rules(Algorithm 3 or Algorithm 4) are globally convergent.

Algorithm 4 The Practical Second-Order Working Set Selection Rule

Compute $\mathbf{q}^{(k)}$ by (14).

if $\boldsymbol{q}^{(k)} \neq \mathbf{0}$ then

i ) Set $\hat{\mathcal{B}}=\mathcal{B}^{(k)}$ and $\mathcal{B}^{(k+1)}=\emptyset$.

ii) Sort the indices set $\{1, \cdots, l\}$ by the values of $\boldsymbol{q}^{(k)}$ in increasing order; Add the first $n_{N}$ indices to form the working set $\mathcal{B}^{(k+1)} ;$ Set $\hat{\mathcal{B}}:=\hat{\mathcal{B}} \backslash \mathcal{B}^{(k+1)}$.

iii) Fill $j \in\left\{i \mid i \in \hat{\mathcal{B}}, 0<\alpha_{i}^{(k)}<C\right\}$, which has the lowest number of consecutive iterations in working set, to $\mathcal{B}^{(k+1)}$ and remove $j$ from $\hat{\mathcal{B}}$ until $\left|\mathcal{B}^{(k+1)}\right|=n_{B}$ or there is no such element.

iv) Fill $j \in\left\{i \mid i \in \hat{\mathcal{B}}, \alpha_{i}^{(k)}=0\right\}$, which has the lowest number of consecutive iterations in working set, to $\mathcal{B}^{(k+1)}$ and remove it from $\hat{\mathcal{B}}$ until $\left|\mathcal{B}^{(k+1)}\right|=n_{B}$ or there is no such element.

v) Fill $j \in\left\{i \mid i \in \hat{\mathcal{B}}, \alpha_{i}^{(k)}=C\right\}$, which has the lowest number of consecutive iterations in working set to $\mathcal{B}^{(k+1)}$ and remove it from $\hat{\mathcal{B}}$ until $\left|\mathcal{B}^{(k+1)}\right|=n_{B}$ or there is no such element.

else

Output $\alpha^{(k)}$ as the optimal solution and terminate the iterations.

end if

Lemma 1. Suppose $\boldsymbol{\alpha}$ is a feasible point of problem (4). $\boldsymbol{\alpha}$ is the KKT point of problem (4) if and only if $\boldsymbol{\ell}(\boldsymbol{\alpha})=\mathbf{0}$.

Proof. Because $\boldsymbol{\alpha}$ is the KKT point of problem (4), from Kuhn-Tucker theorem [18], we know that there exists Lagrange multipliers $\boldsymbol{u}$ and $\boldsymbol{v}$ which satisfies KKT condition: 


$$
\begin{aligned}
F_{i}(\boldsymbol{\alpha})-v_{i}+u_{i}=0, & \forall i=1, \cdots, l \\
v_{i} \alpha_{i}=0, & \forall i=1, \cdots, l \\
u_{i}\left(C-\alpha_{i}\right)=0, & \forall i=1, \cdots, l \\
v_{i}, u_{i} \geq 0, & \forall i=1, \cdots, l
\end{aligned}
$$

Because $\boldsymbol{\alpha}$ is a feasible point of problem (4), there are only the following three kinds of possible value for $\alpha_{i}$ :

(i) If $\alpha_{i} \in(0, C)$, according to the complementary condition $(17 \mathrm{~b})$ and $(17 \mathrm{c})$, we have $v_{i}=u_{i}=0$. Furthermore, from (17a), we know $F_{i}(\boldsymbol{\alpha})=0$. Therefore, $\ell_{i}(\boldsymbol{\alpha})=d_{i} 0=0$.

(ii) If $\alpha_{i}=0$, based on the complementary condition (17c), we have $u_{i}=0$. Furthermore, according to $(17 \mathrm{a})$ and $(17 \mathrm{~d})$, we have $F_{i}(\boldsymbol{\alpha})=v_{i} \geq 0$. Since the feasible set of problem (10) becomes $\left\{d_{i} \mid 0 \leq d_{i} \leq \min \{C, 1\}\right\}$ when $\alpha_{i}=0$, the corresponding optimal solution is $d_{i}=0$. Therefore, $\ell_{i}(\boldsymbol{\alpha})=0 F_{i}(\boldsymbol{\alpha})=0$.

(iii)If $\alpha_{i}=C$, based on the complementary condition (17c), we have $v_{i}=0$. Furthermore, according to (17a) and $(17 \mathrm{~d})$, we have $F_{i}(\boldsymbol{\alpha})=-u_{i} \leq 0$. Since the feasible set of problem (10) becomes $\left\{d_{i} \mid-\min \{C, 1\} \leq d_{i} \leq 0\right\}$ when $\alpha_{i}=0$, the corresponding optimal solution is $d_{i}=0$. Therefore, $\ell_{i}(\boldsymbol{\alpha})=0 F_{i}(\boldsymbol{\alpha})=0$.

Now we prove that if the feasible point of problem (4) $\boldsymbol{\alpha}$ satisfies $\boldsymbol{\ell}(\boldsymbol{\alpha})=\mathbf{0}, \boldsymbol{\alpha}$ must be the KKT point.

For any $i \in\{1, \cdots, l\}$, let $d_{i}^{*}$ denote the optimal solution of problem (10). Since $\ell_{i}(\boldsymbol{\alpha})=F_{i}(\boldsymbol{\alpha}) d_{i}^{*}=0$, we know $F_{i}(\boldsymbol{\alpha})=0$ or $d_{i}^{*}=0$.

(i)If $F_{i}(\boldsymbol{\alpha})=0$, we can choose $u_{i}=v_{i}=0$;

(ii)If $F_{i}(\boldsymbol{\alpha})>0$, we have $d_{i}^{*}=-\alpha_{i}=0$, namely, $\alpha_{i}=0$. Choose $v_{i}=F_{i}(\boldsymbol{\alpha})$ and $u_{i}=0$;

(iii))If $F_{i}(\boldsymbol{\alpha})<0$, we have $d_{i}^{*}=C-\alpha_{i}=0$, namely, $\alpha_{i}=C$. Choose $u_{i}=-F_{i}(\boldsymbol{\alpha})$ and $v_{i}=0$.

It is not difficult to verify that the above chosen value of $\boldsymbol{u}, \boldsymbol{v}$ and $\boldsymbol{\alpha}$ satisfy the KKT condition (17).

Lemma 2. Suppose the kernel function $K(\cdot, \cdot)$ satisfies Mercer condition [16], $\boldsymbol{\alpha}$ is a feasible point of problem (4), then for any $p \in\{1, \cdots, l\}$, we have

$$
Q \operatorname{sub}(\boldsymbol{\alpha}, p) \leq \frac{\ell_{p}(\boldsymbol{\alpha})}{2} \min \left\{1,-\frac{\ell_{p}(\boldsymbol{\alpha})}{2 M C^{2}}\right\},
$$

where, $M=\max _{i=1}^{l}\left\{K_{i, i}+1\right\}$.

Proof. For any index $p \in\{1, \cdots, l\}$, let $\bar{d}_{p}$ denote the solution of problem (10) when $i=p$. For any $t \in[0,1], t \bar{d}_{p}$ stay in the feasible region and $\left|\bar{d}_{p}\right| \leq C$. Furthermore, since kernel function $K(\cdot, \cdot)$ satisfies Mercer condition, we have $K_{p, p} \geq 0$, and $M=$ 
$\max _{i=1}^{l}\left\{K_{i, i}+1\right\} \geq 1$. In all,

$$
\begin{aligned}
\operatorname{Qsub}\left(\boldsymbol{\alpha}^{(k)}, p\right) & \leq \min _{t \in[0,1]}\left\{\bar{d}_{p} F_{p}(\boldsymbol{\alpha}) t+\frac{1}{2} K_{p, p} \bar{d}_{p}^{2} t^{2}\right\} \\
& \leq \min _{t \in[0,1]}\left\{\ell_{p}(\boldsymbol{\alpha}) t+\frac{1}{2} K_{p, p} \bar{d}_{p}^{2} t^{2}\right\} \\
& \leq \min _{t \in[0,1]}\left\{\ell_{p}(\boldsymbol{\alpha}) t+\frac{1}{2}\left(K_{p, p}+1\right) \bar{d}_{p}^{2} t^{2}\right\} \\
& \leq \min _{t \in[0,1]}\left\{\ell_{p}(\boldsymbol{\alpha}) t+\frac{1}{2} M C^{2} t^{2}\right\} \\
& \leq \frac{\ell_{p}(\boldsymbol{\alpha})}{2} t^{*}
\end{aligned}
$$

where, $t^{*}=\min \left\{1,-\frac{\ell_{p}(\boldsymbol{\alpha})}{2 M C^{2}}\right\}$.

Theorem 1. Suppose Suppose the kernel function $K(\cdot, \cdot)$ satisfies Mercer condition [16], $n_{B} \geq n_{N} \geq 1$. Let $\left\{\boldsymbol{\alpha}^{(k)}\right\}$ denote the iterates generated by Algorithm 1 with the working selection rule in Algorithm 3. If $\left\{\boldsymbol{\alpha}^{(k)}\right\}$ contains only finite elements, the last iteration point must be the global optima of problem (4). If $\left\{\boldsymbol{\alpha}^{(k)}\right\}$ contains infinite elements, any accumulation point is a global optima of problem (4).

Proof. If $\left\{\boldsymbol{\alpha}^{(k)}\right\}$ contains finite elements, based on the working selection rule in Algorithm 2, we know the last iteration point must satisfy $\boldsymbol{\ell}\left(\boldsymbol{\alpha}^{(k)}\right)=\mathbf{0}$. From lemma 1 , it must be a KKT point. Hence, we know that $\boldsymbol{\alpha}$ is a KKT point. Hence, we only discuss the situation of infinite iteration points.

Let $\overline{\boldsymbol{\alpha}}$ be any accumulation of the sequence $\left\{\boldsymbol{\alpha}^{(k)}\right\}$. Without loss of generalization, we can assume $\left\{\boldsymbol{\alpha}^{(k)}\right\}$ converge to $\overline{\boldsymbol{\alpha}}$ (this requirement always can be obtained by the proper relabeled of the order of iteration points). Because the feasible region of problem (4) is a bounded closed set in $\Re^{l}$ and the iterates generated by the decomposition algorithm are always feasible, we know that $\overline{\boldsymbol{\alpha}}$ is also a feasible point. Furthermore, the value of $f(\overline{\boldsymbol{\alpha}})$ is a finite number.

Let the index $p$ satisfy

$$
\ell_{p}^{(k)}=\min _{1 \leq i \leq l} \ell_{i}^{(k)}=-\left\|\ell^{(k)}\right\|_{\infty}
$$

Since $n_{N} \geq 1$, index $p$ must be contained in the selected working set $\mathcal{B}^{(k)}$. Hence,

$$
f^{(k+1)}-f^{(k)} \leq Q \operatorname{sub}\left(\boldsymbol{\alpha}^{(k)}, p\right) .
$$

From Lemma 2, we have

$$
\begin{aligned}
f^{(k+1)}-f^{(k)} & \leq \frac{1}{2} \ell_{p}^{(k)} \min \left\{1,-\frac{\ell_{p}^{(k)}}{2 M C^{2}}\right\} \\
& =-\frac{1}{2}\left\|\ell^{(k)}\right\|_{\infty} \min \left\{1, \frac{\left\|\ell^{(k)}\right\|_{\infty}}{2 M C^{2}}\right\}
\end{aligned}
$$


Sum the above formulae from 0 to $s$, we get

$$
\sum_{k=0}^{s}\left\|\ell^{(k)}\right\|_{\infty} \min \left\{1, \frac{\left\|\boldsymbol{\ell}^{(k)}\right\|_{\infty}}{2 M C^{2}}\right\} \leq f^{(0)}-f^{(s+1)} .
$$

Let $s \rightarrow \infty$, we have

$$
\sum_{k=0}^{\infty}\left\|\ell^{(k)}\right\|_{\infty} \min \left\{1, \frac{\left\|\ell^{(k)}\right\|_{\infty}}{2 M C^{2}}\right\} \leq f^{(0)}-f(\overline{\boldsymbol{\alpha}})<+\infty .
$$

Therefore,

$$
\|\boldsymbol{\ell}(\overline{\boldsymbol{\alpha}})\|_{\infty}=\lim _{k \rightarrow+\infty}\left\|\boldsymbol{\ell}\left(\boldsymbol{\alpha}^{(k)}\right)\right\|_{\infty}=0 .
$$

From lemma 1 , we know $\overline{\boldsymbol{\alpha}}$ is the KKT point of problem (4).

Since the kernel function $K(\cdot, \cdot)$ satisfies Mercer condition, problem (4) is a convex problem. Therefore, $\overline{\boldsymbol{\alpha}}$ is a global optima of problem (4).

Lemma 3. Suppose kernel function $K(\cdot, \cdot)$ satisfies Mercer condition [16], a feasible point of problem (4) $\boldsymbol{\alpha}$ is a KKT point if and only if $\boldsymbol{q}(\boldsymbol{\alpha})=\mathbf{0}$.

Proof. Because kernel function $K(\cdot, \cdot)$ satisfies Mercer condition, for all $i \in\{1, \cdots, l\}$, $Q_{i i}>0$. From the definition of $\boldsymbol{q}(\boldsymbol{\alpha})$, we know that $\forall i \in\{1, \cdots, l\}, q_{i}(\boldsymbol{\alpha})$ is the optimal objective function value of $\mathrm{QP}$

$$
\begin{array}{cc}
\min _{d_{i}} & F_{i}(\boldsymbol{\alpha}) d_{i}+\frac{1}{2} Q_{i, i} d_{i}^{2} \\
\text { s.t. } & \max \left\{-1,-\alpha_{i}\right\} \leq d_{i} \leq \min \left\{1, C-\alpha_{i}\right\} .
\end{array}
$$

Let $d_{i}^{*}$ denote the optimal solution of (16), we have $q_{i}(\boldsymbol{\alpha})=d_{i}^{*} F_{i}(\boldsymbol{\alpha})+\frac{1}{2} Q_{i i}\left(d_{i}^{*}\right)^{2}$.

Firstly, let us prove that any KKT point $\boldsymbol{\alpha}$ of problem (4) satisfies $\boldsymbol{q}(\boldsymbol{\alpha})=\mathbf{0}$. Because $\boldsymbol{\alpha}$ is the KKT point of problem (4), from Kuhn-Tucker theorem [18], we know that there exists Lagrange multipliers $\boldsymbol{u}$ and $\boldsymbol{v}$ which satisfies KKT condition:

$$
\begin{aligned}
F_{i}(\alpha)-v_{i}+u_{i}=0, & \forall i=1, \cdots, l ; \\
v_{i} \alpha_{i}=0, & \forall i=1, \cdots, l ; \\
u_{i}\left(C-\alpha_{i}\right)=0, & \forall i=1, \cdots, l ; \\
v_{i}, u_{i} \geq 0, & \forall i=1, \cdots, l .
\end{aligned}
$$

Because $\boldsymbol{\alpha}$ is a feasible point of problem (4), there are only the following three kinds of possible value for $\alpha_{i}$ :

(i) If $\alpha_{i} \in(0, C)$, according to the complementary condition (17b) and (17c), we have $v_{i}=u_{i}=0$. Furthermore, from $(17 \mathrm{a})$, we know $F_{i}(\boldsymbol{\alpha})=0$. Through simple computation, we get that the optimal objective function value of problem (16) is 0 . Therefore, $q_{i}(\boldsymbol{\alpha})=0$.

(ii) If $\alpha_{i}=0$, based on the complementary condition (17c), we have $u_{i}=0$. Furthermore, according to $(17 \mathrm{a})$ and $(17 \mathrm{~d})$, we have $F_{i}(\boldsymbol{\alpha})=v_{i} \geq 0$. Then, the feasible set of problem (16) becomes $\left\{d_{i} \mid 0 \leq d_{i} \leq \min \{C, 1\}\right\}$. Since the symmetric axis of quadratic function (16a) is $-\frac{F_{i}(\boldsymbol{\alpha})}{2 Q_{i i}} \leq 0$, we know the optimal solution is obtained at 
zero, namely $q_{i}(\boldsymbol{\alpha})=0$.

(iii) Similar to the discussion in (ii), we can prove that $d_{i}^{*}=0$ and $q_{i}(\boldsymbol{\alpha})=0$.

Now we prove that if a feasible point of problem (4) $\boldsymbol{\alpha}$ satisfies $\boldsymbol{q}(\boldsymbol{\alpha})=\mathbf{0}, \boldsymbol{\alpha}$ is the KKT point of problem (4).

For any $i \in\{1, \cdots, l\}$, if $q_{i}(\boldsymbol{\alpha})=0$, we know $d_{i}^{*}=0$ or $d_{i}^{*}=-\frac{2 F_{i}(\boldsymbol{\alpha})}{Q_{i i}}$. In fact, if $d_{i}^{*}=-\frac{2 F_{i}(\boldsymbol{\alpha})}{Q_{i i}} \neq 0$, because of the convexity of the feasible region, it is easy to prove that $q_{i}(\boldsymbol{\alpha})<0$, which is contradict to the known fact of $q_{i}(\boldsymbol{\alpha})=0$. Therefore, $d_{i}^{*}=0$. (i) If the symmetric axis of (16a) is $-\frac{F_{i}(\boldsymbol{\alpha})}{2 Q_{i i}}<0$, because $d_{i}^{*}=0$, there is $\alpha_{i}=0$. Set $\left.v_{i}=F_{i}(\boldsymbol{\alpha})\right)>0$ and $u_{i}=0$.

(ii)If the symmetric axis of (16a) is $-\frac{F_{i}(\boldsymbol{\alpha})}{2 Q_{i i}}>0$, because $d_{i}^{*}=0$, there is $\alpha_{i}=C$. Set $\left.u_{i}=-F_{i}(\boldsymbol{\alpha})\right)>0$ and $v_{i}=0$.

(iii)If the symmetric axis of $(16 \mathrm{a})$ is $-\frac{F_{i}(\boldsymbol{\alpha})}{2 Q_{i i}}=0$. We have $\left.F_{i}(\boldsymbol{\alpha})\right)=0$. Set $u_{i}=v_{i}=0$. It can be checked easily that the above chosen $\boldsymbol{u}$ and $\boldsymbol{v}$, together with $\boldsymbol{\alpha}$ satisfy the KKT condition (17).

Theorem 2. Suppose the kernel function $K(\cdot, \cdot)$ satisfies Mercer condition, $n_{B} \geq$ $n_{N} \geq 1$. Let $\left\{\boldsymbol{\alpha}^{(k)}\right\}$ denote the iterates generated by Algorithm 1 with working selection rule in Algorithm 4. If $\left\{\boldsymbol{\alpha}^{(k)}\right\}$ contains only finite elements, the last iteration point must be the global optima of problem (4). If $\left\{\boldsymbol{\alpha}^{(k)}\right\}$ contains infinite elements, any accumulation point is a global optima of problem (4).

Proof. If $\left\{\boldsymbol{\alpha}^{(k)}\right\}$ contains finite elements, the last iteration point must satisfy $\boldsymbol{q}\left(\boldsymbol{\alpha}^{(k)}\right)=$ 0. From lemma 3 , it know that $\boldsymbol{\alpha}$ is a KKT point. Hence, we only discuss the situation of infinite iteration points.

Let $\overline{\boldsymbol{\alpha}}$ be any accumulation point of $\left\{\boldsymbol{\alpha}^{(k)}\right\}$. Without loss of generalization, we can assume $\left\{\boldsymbol{\alpha}^{(k)}\right\}$ converge to $\overline{\boldsymbol{\alpha}}$ (this requirement always can be obtained by the proper relabeled of the order of iteration points). Because the feasible region of problem (4) is a bounded closed set in $\Re^{l}$ and the iterates generated by the decomposition algorithm are always feasible, we know that $\overline{\boldsymbol{\alpha}}$ is also a feasible point. Furthermore, the value of $f(\overline{\boldsymbol{\alpha}})$ is a finite number.

Let the index $p$ satisfy $q_{p}^{(k)}=\min _{1 \leq i \leq l} q_{i}^{(k)}=-\left\|\boldsymbol{q}^{(k)}\right\|_{\infty}$, where the last equality is based on the definition of $\ell_{\infty}$ and the fact of vector $\boldsymbol{q} \leq \mathbf{0}$. Since $n_{N} \geq 1$, index $p$ must be contained in the selected working set $\mathcal{B}^{(k)}$. On the other hand, according to the definition of $\boldsymbol{q}(\boldsymbol{\alpha})$, we know $Q \operatorname{sub}(\boldsymbol{\alpha}, p)=q_{p}(\boldsymbol{\alpha})$. Hence,

$$
f^{(k+1)}-f^{(k)} \leq Q \operatorname{sub}\left(\boldsymbol{\alpha}^{(k)}, p\right)=-\left\|\boldsymbol{q}^{(k)}\right\|_{\infty} .
$$

Sum the above formulae from 0 to $s$,

$$
\sum_{k=0}^{s}\left\|\boldsymbol{q}^{(k)}\right\|_{\infty} \leq f^{(0)}-f^{(s+1)}
$$

Let $s \rightarrow \infty$, we have

$$
\sum_{k=0}^{\infty}\left\|\boldsymbol{q}^{(k)}\right\|_{\infty} \leq f^{(0)}-f(\overline{\boldsymbol{\alpha}})<+\infty
$$


Therefore,

$$
\|\boldsymbol{q}(\overline{\boldsymbol{\alpha}})\|_{\infty}=\lim _{k \rightarrow+\infty}\left\|\boldsymbol{q}\left(\boldsymbol{\alpha}^{(k)}\right)\right\|_{\infty}=0 .
$$

From lemma 3 , we know $\overline{\boldsymbol{\alpha}}$ is the KKT point of problem (4).

Since the kernel function $K(\cdot, \cdot)$ satisfies Mercer condition, problem (4) is a convex problem. Therefore, $\overline{\boldsymbol{\alpha}}$ is a global optima.

\section{Algorithm implementation and numerical experiments}

Experiments are carried out in this section to check the efficiency of our new algorithms. We choose the famous package BSVM $[9]^{2}$ as the baseline. Thanks very much to the authors of BSVM for making their source code public available. In order to emphasize the working set selection rules used, we call the implementation of the Algorithm 3 as LBSVM, and call the implementation of the Algorithm 4 as PQBSVM. Here L means linear and PQ means part of quadratic information is used when selecting the working set.

Besides the working set selection rule, another important component which affects the efficiency of the decomposition algorithm is how to solve the quadratic subproblem (8) at each iteration. According to the structure of the feasible set (8b), we choose projected gradient(PG) algorithm as the default solver for subproblems. However, in some difficult cases, such as when the condition number for the Hessian matrix of (8a) is large, PG method may fail to find the solution satisfied the required accuracy in a given number of iterations. In this difficult case, we call the interior point method(IPM) to solve the QP subproblem (8).

The new algorithms is implemented in $\mathrm{C}$ and compile by gcc-4.1.3 with -O3 option. The source code is available upon request from the authors. The underlying PG solver is a modification of PG solver DFGPM in GPDT(Zanni, Serafini and Zanghirati, 7/2/2007 [23]). For the IPM Solver, we use OOQP (Gertz and Wright, 3/9/2008 [6]). A cache is maintained by the Least-Recent-Used principle to store part of the kernel elements which have been calculated.

The cache size for both BSVM,LMSVM and PQBSVM is the default size of BSVM: $100 \mathrm{M}$. The termination accuracy for both algorithms is $10^{-3}$. We selected 15 public data sets from text and image classification, hand-written recognition, bioinformation and economics as test problems. ${ }^{3}$ We summarize the data statistics in Table 1.

Gauss kernel $K(\boldsymbol{x}, \boldsymbol{y})=e^{-\gamma\|\boldsymbol{x}-\boldsymbol{y}\|^{2}}$ are used in the experiment. Model parameter $\mathrm{C}$ and kernel parameter $\gamma$ are selected by the parameter tuning method proposed in [7]. For dataset whose size is larger than 10,000, we random take 5,000 samples as the tuning data set. Otherwise, the whole data set is used. Then the tuning dataset is divided into two parts: $70 \%$ for training and $30 \%$ for validation. The space we explore

\footnotetext{
${ }^{2}$ BSVM can be downloaded from http://www.csie.ntu.edu.tw/ cjlin/bsvm/

3 "ijcnn1" is from the International Joint Conference on Neural Networks Competition; "real-sim" is downloaded from http://www.cs.umass.edu/ mccallum/code-data.html; "news20", "rcv1" and "webpage" are down loaded from http://www.csie.ntu.edu.tw/ cjlin/libsvmtools/datasets/. All the others are from the UCI repository [1].
} 
Table 1: Dataset Statistic and Optimal Parameters

\begin{tabular}{llllcc}
\hline name & \#train & \#test & \#dim. & $\mathrm{C}$ & $\gamma$ \\
\hline adult & 32561 & 16281 & 123 & $2^{7}$ & $2^{-10}$ \\
\hline australian & 690 & $\mathrm{~N} / \mathrm{A}$ & 14 & $2^{-1}$ & $2^{-2}$ \\
\hline breast-cancer & 683 & $\mathrm{~N} / \mathrm{A}$ & 10 & $2^{-2}$ & $2^{-3}$ \\
\hline diabetes & 768 & $\mathrm{~N} / \mathrm{A}$ & 8 & $2^{2}$ & $2^{-5}$ \\
\hline german & 1000 & $\mathrm{~N} / \mathrm{A}$ & 24 & $2^{7}$ & $2^{-10}$ \\
\hline heart & 270 & $\mathrm{~N} / \mathrm{A}$ & 13 & $2^{0}$ & $2^{-6}$ \\
\hline ijcnn1 & 49990 & 91701 & 22 & $2^{5}$ & $2^{0}$ \\
\hline ionosphere & 351 & $\mathrm{~N} / \mathrm{A}$ & 34 & $2^{4}$ & $2^{-5}$ \\
\hline liverdisorder & 345 & $\mathrm{~N} / \mathrm{A}$ & 6 & $2^{6}$ & $2^{-2}$ \\
\hline mushrooms & 8124 & $\mathrm{~N} / \mathrm{A}$ & 22 & $2^{-1}$ & $2^{-3}$ \\
\hline news20 & 19996 & $\mathrm{~N} / \mathrm{A}$ & 1255191 & $2^{5}$ & $2^{-5}$ \\
\hline rcv1 & 20242 & 677399 & 47236 & $2^{1}$ & $2^{0}$ \\
\hline real-sim & 72309 & $\mathrm{~N} / \mathrm{A}$ & 20958 & $2^{5}$ & $2^{-1}$ \\
\hline sonar & 208 & $\mathrm{~N} / \mathrm{A}$ & 60 & $2^{0}$ & $2^{-2}$ \\
\hline webpage & 49749 & 14951 & 300 & $2^{10}$ & $2^{-10}$ \\
\hline
\end{tabular}

for optimal $\mathrm{C}$ and $\gamma$ are in the set $\left\{2^{-2}, 2^{-1}, \cdots, 2^{11}, 2^{12}\right\}$ and $\left\{2^{-10}, 2^{-9}, \cdots, 2^{3}, 2^{4}\right\}$, respectively. The chosen best kernel and model parameters are given in Table 1.

We increase the size of the working set from 10 to 1280 , and we recorded the corresponding results in Table 2. Since LBSVM,PQBSVM and BSVM consider the same model problem (2) and same termination accuracy is used. We do not list prediction accuracy, which is the same for each comparisons presented in Table 2. The column "\#WS" represents the size of working set. The column "Time" represents the training time. The shortest training time for each case is marked in bold letter. And in every case, we marked the shortest training time for different working set size in italics. The column "Iter." is the number of iterations. From Table 2, we can see that, the performance of the new algorithms is at least as good as BSVM. In particular, the PQBSVM algorithm have reached the optimum in most circumstances for all test questions.

Another thing needs to be inspected is the relationship between the training time, the number of iterations and the size of working set. Figure 1 shows the situation for the four test problems: ijcnn1,news20,rcv1 and real-sim, which gives us some intuitive explanation. The number of iterations decreases with the size of the working set. The training time drops with the increasing of working set size at the beginning because of the reduction of iteration numbers. However, when the working set becomes too large, the time for solving the QP subproblem will dominate the training time and the training time starts to increase gradually. This phenomenon meets the general characteristics of decomposition based SVM training algorithm [23]. Perhaps we can exploit and benefit from more larger working set size with more robust inner QP solvers. This topic is one of our future works. 
Table 2: Comparisons of BSVM,LBSVM and PQBSVM

\begin{tabular}{|c|c|c|c|c|c|c|c|}
\hline \multirow{2}{*}{ Dataset } & \multirow{2}{*}{$\# \mathrm{WS}$} & \multicolumn{2}{|c|}{ Lin's BSVM } & \multicolumn{2}{|c|}{ LBSVM } & \multicolumn{2}{|c|}{ PQBSVM } \\
\hline & & time $(\mathrm{s})$ & iter. & time $(\mathrm{s})$ & iter. & time $(\mathrm{s})$ & iter. \\
\hline \multirow{8}{*}{ adult } & 10 & 344.35 & 14064 & 279.17 & 15548 & 214.35 & 7163 \\
\hline & 20 & 377.81 & 6475 & 247.68 & 7728 & 198.48 & 3985 \\
\hline & 40 & 434.82 & 2885 & 186.01 & 3077 & 159.49 & 1627 \\
\hline & 80 & 643.92 & 1204 & 161.46 & 863 & $155.4^{2}$ & 754 \\
\hline & 160 & 1015.46 & 621 & 185.08 & 396 & 177.66 & 405 \\
\hline & 320 & 1517.47 & 387 & 252.77 & 261 & 246.02 & 202 \\
\hline & 640 & 3831.03 & 290 & 749.15 & 115 & 753.77 & 149 \\
\hline & 1280 & 22390.78 & 197 & 2781.42 & 99 & 1100.64 & 35 \\
\hline \multirow{8}{*}{ ijcnn1 } & 10 & 650.7 & 22794 & 945.16 & 58590 & 356.37 & 12601 \\
\hline & 20 & 671.26 & 10009 & 724.65 & 14464 & 301.37 & 5204 \\
\hline & 40 & 685.16 & 4049 & 512.29 & 4299 & 269.87 & 2302 \\
\hline & 80 & 657.55 & 1600 & 436.45 & 1559 & 239.83 & 913 \\
\hline & 160 & 574.88 & 593 & 363.92 & 546 & 196.7 & 350 \\
\hline & 320 & 528.47 & 209 & 284.28 & 199 & 163.32 & 139 \\
\hline & 640 & 596.63 & 67 & 372.48 & 60 & 256.61 & 52 \\
\hline & 1280 & 6126.54 & 32 & 807.66 & 27 & 797.31 & 24 \\
\hline \multirow{8}{*}{ mushrooms } & 10 & 7.31 & 3160 & 3.37 & 902 & 0.95 & 145 \\
\hline & 20 & 7.46 & 1642 & 3.53 & 486 & 1.19 & 98 \\
\hline & 40 & 8.08 & 917 & 3.53 & 248 & 1.38 & 59 \\
\hline & 80 & 8.96 & 411 & 4.07 & 126 & 1.59 & 33 \\
\hline & 160 & 10.41 & 168 & 4.66 & 65 & 2.34 & 22 \\
\hline & 320 & 14.47 & 65 & 7.32 & 29 & 3.59 & 13 \\
\hline & 640 & 33.21 & 28 & 20.34 & 14 & 8.62 & 6 \\
\hline & 1280 & 141.85 & 14 & 59.8 & 8 & 30.36 & 4 \\
\hline \multirow{8}{*}{ news20 } & 10 & 2830.06 & 8070 & 3246.46 & 5999 & 1684.09 & 2734 \\
\hline & 20 & 2877.79 & 4088 & 3234.45 & 3013 & 1751.96 & 1383 \\
\hline & 40 & 2896.77 & 2054 & 3296.57 & 1484 & 1692 & 685 \\
\hline & 80 & 2798.33 & 994 & 3190.79 & 742 & 1736.11 & 344 \\
\hline & 160 & 2773.31 & 493 & 3275.61 & 372 & 1691.63 & 175 \\
\hline & 320 & 3463.19 & 316 & 3476.86 & 192 & 1831.32 & 92 \\
\hline & 640 & 3049.63 & 129 & 3593.00 & 96 & 1973.89 & 48 \\
\hline & 1280 & 3280.09 & 60 & 4412.36 & 48 & 2527.88 & 24 \\
\hline \multirow{8}{*}{ rcv1 } & 10 & 1380.28 & 8572 & 710.2 & 7681 & 314.08 & 3207 \\
\hline & 20 & 1435.63 & 4456 & 668.26 & 3839 & 321.37 & 1645 \\
\hline & 40 & 1467.83 & 2288 & 676.63 & 1921 & 328.41 & 836 \\
\hline & 80 & 1489.84 & 1160 & 661.53 & 947 & 324.01 & 423 \\
\hline & 160 & 1426.52 & 547 & 687.94 & 472 & 335.43 & 215 \\
\hline & 320 & 1475.34 & 285 & 696.94 & 231 & 342.85 & 107 \\
\hline & 640 & 1817.97 & 167 & 742.63 & 114 & 367.25 & 53 \\
\hline & 1280 & 2432.59 & 89 & 1061.62 & 60 & 484.07 & 27 \\
\hline
\end{tabular}




\begin{tabular}{c|l|l|l|l|l|l|l}
\hline \multirow{2}{*}{ Dataset } & \multirow{2}{*}{$\#$ WS } & \multicolumn{2}{|c|}{ Lin's BSVM } & \multicolumn{2}{c|}{ LBSVM } & \multicolumn{2}{c}{ PQBSVM } \\
\cline { 2 - 8 } & & time(s) & iter. & time(s) & iter. & time(s) & iter. \\
\hline \multirow{5}{*}{ real-sim } & 10 & 3570.9 & 14778 & 2092.14 & 12331 & $\mathbf{8 3 3 . 2 3}$ & 4617 \\
\cline { 2 - 8 } & 20 & 3620.44 & 7475 & 2018.87 & 6337 & $\mathbf{8 5 0}$ & 2419 \\
\cline { 2 - 8 } & 40 & 3676.19 & 3794 & 2056.29 & 3190 & $\mathbf{8 6 9 . 8 2}$ & 1249 \\
\cline { 2 - 8 } & 80 & 3603.62 & 1866 & 2151.53 & 1608 & $\mathbf{8 8 4 . 7 4}$ & 648 \\
\cline { 2 - 8 } & 160 & 3549.23 & 908 & 2204.45 & 817 & $\mathbf{8 6 3 . 0 2}$ & 316 \\
\cline { 2 - 8 } & 320 & 4263.11 & 549 & 2302.09 & 408 & $\mathbf{9 3 0 . 3 7}$ & 163 \\
\cline { 2 - 8 } & 640 & 4346.47 & 261 & 4611.39 & 205 & $\mathbf{1 8 6 6 . 3 1}$ & 82 \\
\cline { 2 - 8 } & 1280 & 4482.8 & 116 & 5150.1 & 103 & $\mathbf{2 1 2 7 . 2 4}$ & 42 \\
\hline \multirow{5}{*}{ webpage } & 10 & 597.79 & 64591 & 2443.66 & 120330 & $\mathbf{4 1 2 . 4 1}$ & 12939 \\
\cline { 2 - 8 } & 20 & 728.79 & 34362 & 1654.42 & 40621 & $\mathbf{4 3 4 . 1 9}$ & 6960 \\
\cline { 2 - 8 } & 40 & 915.8 & 15449 & 1422.96 & 16380 & $\mathbf{4 2 6 . 4 2}$ & 2668 \\
\cline { 2 - 8 } & 16 & 1096.38 & 5517 & 1142.26 & 6138 & $\mathbf{3 1 0 . 9 1}$ & 1263 \\
\cline { 2 - 8 } & 1299.64 & 1951 & 924.75 & 1989 & $\mathbf{2 2 2 . 4 5}$ & 402 \\
\cline { 2 - 8 } & 320 & 976.65 & 403 & 774.38 & 403 & $\mathbf{1 5 6 . 4 6}$ & 100 \\
\cline { 2 - 7 } & 640 & 760.68 & 85 & 2219.40 & 245 & $\mathbf{3 7 4 . 9 4}$ & 45 \\
\cline { 2 - 7 } & 1280 & 2167.48 & 32 & 51920.36 & 1410 & $\mathbf{1 0 2 8 . 9}$ & 22 \\
\hline
\end{tabular}

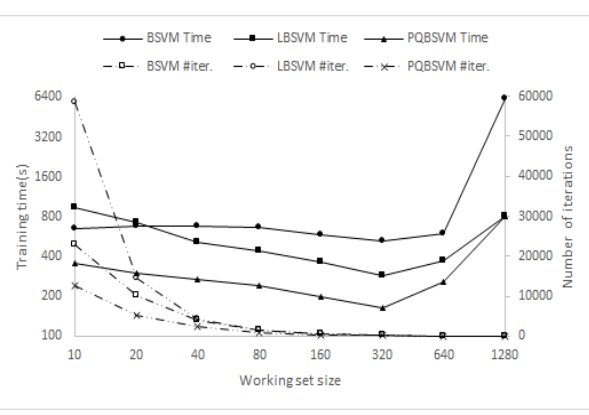

(a) ijcnn1

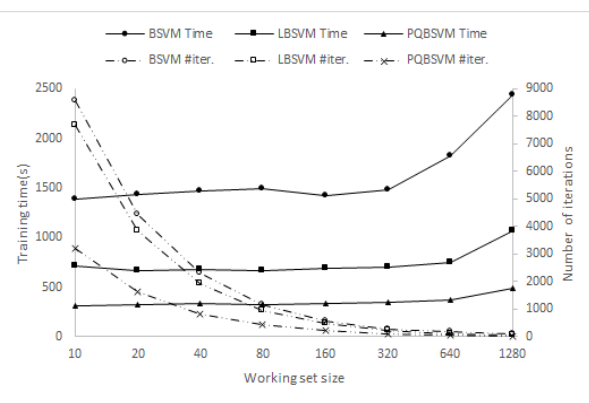

(c) rcv1

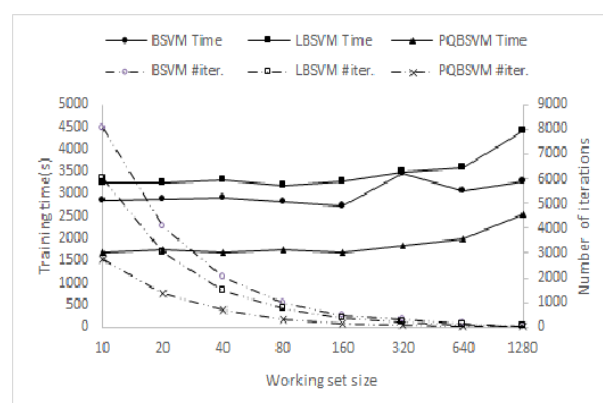

(b) news20

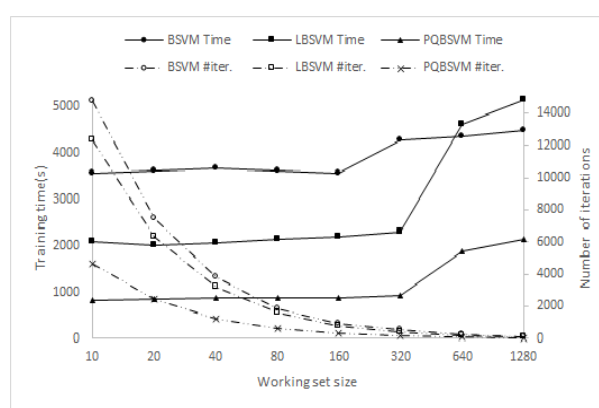

(d) real-sim

Figure 1: Working set size to training time and number of iterations. 


\section{Conclusions and Future Work}

We propose two new decomposition algorithms for training bound-constrained SVMs in this paper. These two methods are particularly suitable for trainning nonlinear SVMs. In the process of selecting working set, when we only use first order information of the objective function, we get a first-order decomposition algorithm. To make the working set selection strategy more effective, we utilize part of second order information and first order information together to choose the working set, which results in Algorithm 4. To the best of our knowledge, this is the first non-SMO decomposition algorithm which can use second order information for selecting working set. Both algorithms are proved to be globally convergent. PG method and IPM are combined together to solve the quadratic subproblems. Experiments on benchmark problems validate the efficiency of the new algorithms.

One of the our ongoing work currently is further improving the efficiency of our implementation. We plan to include other types of QP solver for the subproblem solution, which can handle very bad scale problems better. We also consider introducing more careful working set selection strategy to reduce iteration numbers. Last but not least, a careful shrinking strategy should be used to further reduce the number of kernel evaluations.

\section{Acknowledgement}

This work was supported by the National Natural Science Foundation of China(Grant No. 11201472, 11331012, 71110107026).

\section{References}

[1] Asuncion A., Newman D.,UCI Machine Learning Repository, University of California, Irvine, School of Information and Computer Sciences (2007) http://www. ics.uci.edu/ mlearn/MLRepository.html.

[2] Boser B., Guyon I., Vapnik V.A., A training algorithm for optimal margin classifiers, Proceedings of the 5th Annual ACM Workshop on Computational Learning Theory, ACM Press, 1992, 144-152.

[3] Bottou L., Stochastic gradient descent examples, 2007, http://leon.bottou. org/projects/sgd.

[4] Cortes C., Vapnik V., Support-vector networks, Machine Learning, 20, 3, 1995, 273-297.

[5] Franc V., Sonnenburg S., Optimized cutting plane algorithm for support vector machines, ICML 08: Proceedings of the 25th international conference on Machine learning, ACM Press 2008, 320-327. 
[6] Gertz E., Wright S., Object-oriented software for quadratic programming, ACM Transactions on Mathematical Software, 29, 2001, 58-81.

[7] Hsieh C., Chang K., Li C.J., A comparison of methods for multi-class support vector machines, IEEE Transactions on Neural Networks, 13, 2002, 415-425.

[8] Hsieh C., Chang K., Lin C.J., Keerthi S., Sundararajan S., A dual coordinate descent method for large-scale linear SVM, Proceedings of the 25th international conference on Machine learning, ACM, 2008, 408-415.

[9] Hsu C.W., Lin C.J., A simple decomposition method for support vector machines, Machine Learning, 46, 1-3, 2002, 291-314.

[10] Joachims T., SVM ${ }^{\text {light }}$, http://svmlight.joachims.org/.

[11] Joachims T., Training linear SVMs in linear time, ACM SIGKDD International Conference On Knowledge Discovery and Data Mining, 2006, 217-226.

[12] Joachims T., Finley T., Yu C.N., Cutting-plane training of structural svms, Machine Learning, 77, 1, 2009, 27-59.

[13] Joachims T., Yu C.N., Sparse kernel svms via cutting-plane training, Machine Learning, Special Issue for European Conference on Machine Learning, 76, 2-3, 2009, 179-193.

[14] Mangasarian O., Musicant D., Successive overrelaxation for support vector machines, IEEE Transactions on Neural Networks, 10, 5, 1999, 1032-1037.

[15] Mangasarian O., Musicant D., Lagrangian support vector machines, Journal of Machine Learning Research, 1, 2001, 161-177.

[16] Mercer J., Functions of positive and negative type and their connection with the theory of integral equations, Philosophical Transactions of the Royal Society of London, 1909.

[17] Shalev-Shwartz S., Singer Y., Srebro N., Cotter A., Pegasos: Primal Estimated sub-Gradient Solber for SVM, Mathematical Programming, 127, 1, 2011, 3-30.

[18] Sun W.Y., Yuan Y.X., Optimization Theory and Methods: Nonlinear Programming, Springer, New York, USA, 2006.

[19] Frieß T., Cristianini N., Campbell C., The kernel-adatron algorithm: a fast and simple learning procedure for support vector machines, Proceedings of the Fifteenth International Conference on Machine Learning, Morgan Kaufmann Publishers, 1998.

[20] Vapnik V., The Nature of Statistical Learning Theory, Springer-Verlag New York, Inc., New York, NY, USA 1995.

[21] Vapnik V., Statistical Learning TheoryStatistical Learning Theory, WileyInterscience, September, 1998. 
[22] Yuan G.X., Ho C.H., Lin C.J., Recent Advances of Large-scale Linear Classification, Proceedings of the IEEE, 100, 2012, 2584-2603.

[23] Zanni L., Serafini T., Zanghirati G., Parallel software for training large scale support vector machines on multiprocessor systems, Journal of Maching Learning Research, 7, 2006, 1467-1492.

[24] Osuna E., Freund R., Girosi F., Training support vector machines: An application to face detection, Proceedings of IEEE Computer Society Conference on Computer Vision and Pattern Recognition, 1997, 276-285.

[25] Arnosti N.A., Kalita J.K., Cutting Plane Training for Linear Support Vector Machines, IEEE Transactions on Knowledge and Data Engineering, 25, 2013, 1186-1190.

[26] Platt J.-C., Fast training of support vector machines using sequential minimal optimization, in: B. Schölkopf, C.J.C. Burges, A.J. Smola(Eds.), Advances in kernel methods-support vector learning, MIT press, 1999, 185-208.

[27] Joachims T., Making large-scale SVM learning practical, in: B. Schölkopf, C.J.C. Burges, A.J. Smola(Eds.), Advances in kernel methods-support vector learning, Cambridge, MA, MIT press, 1998.

[28] Saunders C., Stitson M.O., Weston J., Bottou L., Schölkopf B., Smola A., Support vector machine reference manual, Egham, UK, Royal Holloway, University of London, Technical Report, NO.CSD-TR-98-03, 1998.

[29] Tian Y.J., Qi Z.Q., Ju X.C., Shi Y., Liu X.H., Nonparallel support vector machines for pattern classification, IEEE Trans. Cybernetics, 44, 7, 2013, 1067-1079.

[30] Qi Z., Tian Y., Shi Y., Successive Overrelaxation for Lapiacian Support Vector Machine, IEEE Transaction on Neural Networks and Learning System, DOI: 10.1109/TNNLS.2014.2320738, 2014.

[31] Qi Z., Tian Y., Shi Y., Structural Twin Support Vector Machine for Classification, Knowledge-Based Systems, 43, 2013, 74-81.

Received 14.11.2014, accepted 26.01.2015 